\title{
20 More resource efficiency in production and products
}

\author{
Digitalisation supports industry \\ and trades
}

Peter Jahns

\section{Introduction}

In 1992, The Global Earth Summit of the United Nations in Rio advocated for cross-cutting sustainability strategies to meet the unrelenting challenges of sustainable development.

In 2002, Germany adopted a National Sustainability Strategy (Bundesregierung 2016). By 2017, fifteen (15) out of sixteen (16) of the Federal States of Germany, including North Rhine-Westphalia (NRW), had adopted regional strategies to address the challenges of sustainable development (Müller \& Reutter 2017).

North Rhine-Westphalia is the most populated federal state in Germany and a central industrial and economic region (Müller \& Reutter 2017). Thus, in 1998, the Ministry for the Environment in North Rhine-Westphalia established the Effizienz-Agentur NRW (EFA) with the principal objective of improving the competitiveness of Small and Medium-sized Enterprises (SMEs) by supporting a strategy of sustainable growth (Jahns 2018).

As per the NRW.INVEST GmbH, Knowledgebase NRW (2018), SMEs in North-Rhine Westphalia are the backbone of the economy. Currently, there are 712,100 SMEs in North-Rhine Westphalia and accounting for 78.8 per cent of employment. Out of this total, 12,000 are small and medium-sized manufacturing companies (20 to 500 employees). Furthermore, there are 19,000 craft enterprises with resource efficiency potential. The majority of all training in North-Rhine Westphalia provided by EFA is for these SMEs. The SME sector is, thus, the focus of EFA (Jahns 2018; Bliesner, Liedtke \& Rohn 2014).

The Effizienz-Agentur NRW (EFA) has been operating for the past twenty (20) years (Jahns 2018). Jahns indicates that the EFA supports manufacturing companies in North-Rhine Westphalia with advice concerning resource efficiency so that products and processes are made more efficiently to reduce costs and adverse effects on the environment. Jahns further indicates that the company offers support for the financing and implementation of research and development projects and investments in the form of financial advice. Additionally, EFA provides network and knowledge transfer opportunities, information about training courses and workshops, with the aim of increasing resource efficiency and competitiveness in SMEs. 
Raw material and material costs represent the most significant proportion of costs for manufacturing companies in Germany. Its share of the total cost of manufacturing is about 42 per cent. Whereas the average Personnel and energy are 18 per cent and two per cent, respectively. It therefore stands to reason to pay careful attention during production and take care when dealing with these natural resources. The focussing on the subject of 'energy' which is unfortunately seen all too often in public discussions in industry and trade does not go far enough. Reducing the cost of raw materials and other materials is the key to separating resource consumption and growth. Efficiency is a decisive factor in this. As far as resource-efficient production is concerned, the focus should be on natural resources. It is, therefore, logical to emphasis the term 'resource efficiency' since the operational goals of EFA are to reduce the use of materials and energy.

EFA is working on concrete projects with small and medium-sized enterprises that allow it (EFA) to create products with less energy and primary resources (Jahns 2018; Kaiser 2018; European Environment Agency 2016). The priority is to avoid waste from the get-go and not wait to start thinking about it in the future. The idea is to prevent waste before it is produced.

The purpose of the chapter is to share some best ways to implement cleaner production and resource efficiency practices that sustainably support industry and trades through digitalisation. The chapter contributes to the conversation on resource efficiency by linking both individual and organisational measures of success with producing more with less energy and primary resources. The remainder of the chapter proceeds as follows. The next section provides a brief description of the Effizienz-Agentur NRW (EFA) Consulting Offer, which is a guide to more resource efficiency. Section two illustrates results from the agency's consultancy practice during a period in which digital networking is becoming the standard. Section three focuses on three cases to illustrate the importance of digitalisation for manufacturing companies, which has been discussed in Germany under the term 'Industry 4.0'. The final section is the conclusion.

\section{The Effizienz-Agentur NRW consulting offer - guide to more resource efficiency}

EFA's consulting offer for more resource efficiency goes through a threepronged process, namely, need for action, initial consultation and solutions (Jahns 2018). Figure 20.1 depicts the three-pronged process.

The three-pronged process, as described by (Jahns 2018) is described in more detail in the following sections.

\section{Need for action}

EFA works with clients to systematically determine needs that ought to be addressed. The need for action assesses gaps between current conditions and improved conditions of performance. 


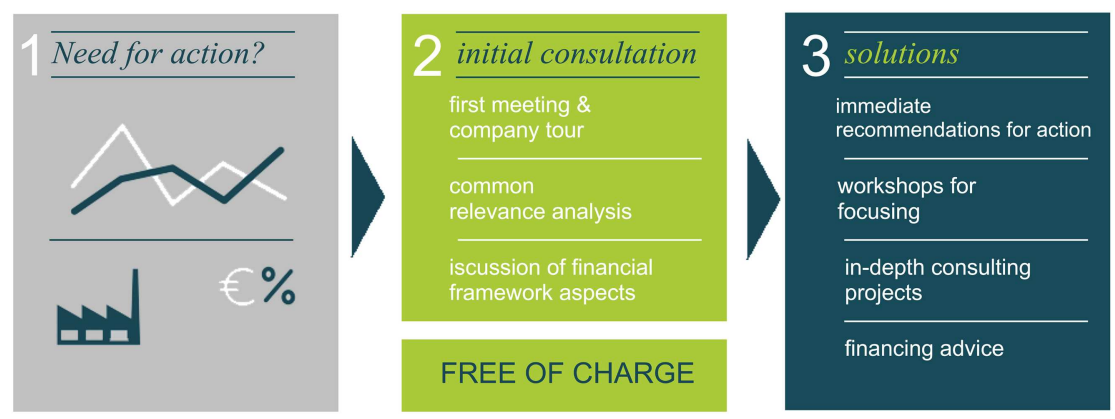

Figure 20.1 EFA consulting - guide to more resource efficiency.

\section{Initial consultation}

The Needs Assessment is a process that helps EFA to understand the clients' needs, motivations, and behaviours - what they do, how they do it, and why. The assessment begins with an initial consultation meeting with the client, which is followed by a company tour. It is an iterative process where common relevance analysis conducted. The common relevance analysis includes compiling and analysing data on trends through interviews, surveys and focus group discussions. After this, EFA discusses a financial framework with the client to set policies, procedures, regulations and standing orders to ensure that public funds are appropriately taken care of. All of these are done at no cost to clients.

\section{Solutions}

Immediate recommendations for action, workshops for focusing, in-depth consulting projects and financing advice are then offered.

\section{Conservation of resources and digitalisation = 'Resource Efficiency 4.0'}

Industry 4.0 represents the digital networking of industrial processes, starting with the acquisition of raw materials through to the recycling of the products at the end of their service life, i.e. in the sense of a circular economy (Jahns 2018; Müller, Buliga \& Voigt 2018). Industry 4.0 solutions can be completely automated and without human influence or partially automated cooperation between man and machine. The automation, which is accompanied by new 
manufacturing and working methods, will also fundamentally change our way of living and consuming in a digital society. Classic mass-produced goods are being replaced with products which are more individualised and flexible production processes. Small batches and even the manufacture of individual items are becoming more of the standard. Web-based process control systems and networked sensor systems in the sense of Industry 4.0 are allowing companies to record their processes more accurately and individually control them - even to the extent of autonomous factories. If information is available in real-time, individual production processes and entire value creation chains can be controlled in such a way that optimum use can be made of capacities. The productivity of the German economy could increase by almost twelve per cent by 2025 , merely due to additional value creation. At the same time, the chances of avoiding wastage are increasing thanks to requirements-based production.

The 'digital transformation' is therefore associated with significant upheavals, disruptions and new challenges, particularly for manufacturing companies. Existing business models are being questioned, products are disappearing from the market, and services are increasing in importance. While a wide range of new possibilities, offered by digitalisation, is proceeding concurrently with a trend towards the individualisation of production and products, the development of new business areas and business models are also emerging.

The manufacturing companies are operating in this conflict area. Particularly the 12,000 small and medium-sized manufacturing companies (20 to 500 employees) and the approximately 19,000 handicraft businesses with enormous potential for conserving resources have little capacity outside of everyday businesses to fundamentally and continually occupying themselves with this strategically important topic of digitalisation and resource conservation in the sense of 'Resource Efficiency 4.0'. The digitalisation and resource conservation offer Effizienz-Agentur NRW an opportunity to provide advisory support.

\section{Resource Efficiency 4.0 - Digitalisation as a tool}

The goal of EFA, i.e. helping companies on the way to more resource-efficient manufacturing, has not changed because of digitalisation. However, that which has changed a great deal for the companies seeking advice are their possibilities about planning and controlling production processes, and adapting products or services to the individual needs of the customer. All action approaches for supporting the companies with a more efficient process design in the digitalisation era have been subsumed into the category of 'Resource Efficiency 4.0' at EFA since 2018. It is crucial to use digitalisation as a useful tool for reducing material and energy consumption and not as an end in itself. Above all, Resource Efficiency 4.0 is an instrument which helps companies with their work. As with all tools, the intention with which you use it is the decisive factor. It is a case of keeping an eye on the 'resource efficiency' indicator. 


\section{Peter Jahns}

The digitalisation of a production line is not a cure-all. The experience gained in the initial 'Industry 4.0' projects in foundries and surface refining companies has shown how meaningful it is to create full process transparency through a resource efficiency analysis. The companies obtain the required transparency regarding the respective use of the material and the associated $\mathrm{CO}_{2}$ emissions. This data forms the basis for introducing or adopting ERP systems. In this way, the decisive success indicators for manufacturing in a more competitive way can first be determined, and resource conservation improved by taking technical measures. On this basis, the competitiveness of the company can be further increased through digitalising production: This approach comes under the expression 'Resource Efficiency 4.0'.

\section{Operational areas of activity}

A company is, usually, confronted by four fields of activity within the scope of providing its services, namely: order clarification, order processing, calculation and the future of the company (see Figure 20.2). The first three fields of activity can be regarded as being of equal importance, whereby the latter area, the future of the company, is strategically important and not a part of everyday business. For small and medium-sized companies, in particular, there is a severe risk of this critical area of activity being taken over by day-to-day business and fading into the background.

\section{ORDER CLARIFICATION}

The customer's requirements and expectations must first be clarified before manufacturing begins. In this way, the manufacturing of erroneous or defective

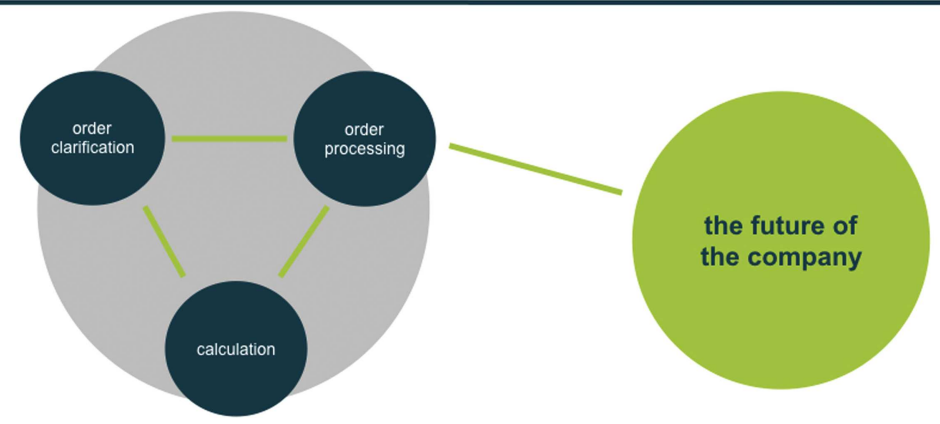

Figure 20.2 Resource Efficiency 4.0 - Fields of activity. 
products due to misunderstandings or inaccurate specifications can be avoided right from the start. As banal as this requirement might sound: consultancy practice has shown that following up, often, does not take place with sufficient diligence in this case, or the customer is promised services by the sales department, for example, which cannot be provided.

The order description at least contains the subject, delivery date and price, the quantity and the delivery location, of course. Depending on the type of order, other requirements may be added concerning commissioning, for example. The order description must be stored on the company's central data management system (digital) in such a way that all employees can access the information that is relevant to them if required.

The company procurement department must be involved during order clarification. The reason is that it is impossible to make a meaningful statement about delivery dates without knowledge of part availability (takes place by comparing with company stock levels) and the delivery times of required materials (raw materials, semi-finished products, bought-in parts). The order data cannot be deemed complete until part lists can be generated on the existing data basis.

ORDER PROCESSING

Order clarification is followed by order processing. In particular, it includes planning, optimisation, visualisation and implementation of all orders using a central data management system. The data basis of these sub-steps is identical to that which is used during order clarification, and should ideally be carried out using the same data system (software program) and therefore without media or system disruption, which minimises transmission errors.

The customer order is organised into numerous work and process steps, and the required documents (e.g. company orders or 'routing slips') are generated automatically. A problem-free and complete flow of information is the basis for stable processes. Through automatic analysis of the existing data, it is possible to make the implicitly present process knowledge transparent and therefore usable for improvement processes (process mining), for example.

A smoothly functioning enterprise resource management system is a prerequisite for interruption-free production. As well as incoming and outgoing goods, enterprise resource management also includes the warehouse system and shipping. Central recording of the workflows (booking in and out of deliveries, assignment of defined storage locations) in the data management system provides information about stock levels 'in real-time'. Automated provision of materials is also a part of the scope of work of a contemporary enterprise resource management system and takes the strain off the employees in the production area. Another advantage: Programcontrolled material management makes an automatic inventory and stock planning and targeted stock reduction possible to counteract overstocking and the resulting disposals. 


\section{Peter Jahns}

Production takes place based on manufacturing plans, which are ideally generated automatically after entering the required data. The definition of the exact system and machine configuration is the task of the production planning and control (PPS) department, which represents the core of a manufacturing company. Here too, digital support from ERP systems, for example, is vital. The production process is monitored and controlled using automatic machine data recording. The efficiency and utilisation of production can be tracked without problems by accurately defining and documenting setup times, the start of orders and the completion of orders.

\section{CALCULATION}

The digital connection of machines and systems to the data managing system makes cause-based cost transparency possible concerning orders, products and processes. In this way, it is comparatively easy to check the calculation after processing a request based on actual person-hours, machine run times and material consumption values, and determine whether the assumptions that were made during pre-calculation were correct. In the event of deviations, more accurate assumptions can be used as the basis for a quote when placing future orders.

Costs, particularly material and energy costs, should be assigned in a causerelated way. After all, the material costs are the most significant cost block in the processing industry. To keep the proportion of general costs as low as possible, cost centres should be analysed and redefined if necessary. Personnel costs must be transferred to individual cost centres. The recording of quantity throughput via cost centres is desirable. Write-offs, room costs, maintenance, energy, operating materials and auxiliary materials must be taken into consideration during the determination of hourly machine rates. Overhead charge rates must be updated at regular intervals.

Cause-related cost assignment is made possible by connecting individual machines (machine data recording [MDE]) and also integrating a higher-order data architecture (operational data recording [BDE]), (Management Executive Systems [MES]) in the ERP system. Exact knowledge of the cost structure puts the business operator in a position to reduce manufacturing costs in a targeted way using efficiency measures and to remove products from the product range in good time.

\section{FUTURE OF THE COMPANY}

The question of the company's future does not come up every day, but it is essential for the company management to also deal with social changes continue to be able to anticipate the effects on company operations in good time. Against the background of the lack of global resources, it is logical to realise that resource efficiency is a strategic element of company development. Continuous and long-term company development can only be 
achieved if the principles of process and resource efficiency are taken into consideration. It is, therefore, advisable to adapt to market changes under resource criteria.

Digital networking is presenting companies with additional challenges. Many processes are easier to comprehend and process because information can be shared quickly. In the same way that trading on the Internet is changing retail structures, production locations that are connected via the Internet will revolutionise manufacturing procedures. Anyone who does not keep a permanent eye on their business environment and researches ways of opening up new business areas is otherwise at risk of losing their market position.

To be able to react to changes quickly, the product development process should have a flexible design. Besides, the topic of resource consumption must always be taken into consideration since 80 per cent of the effects on the environment, and the cost of a product is already defined at the design stage. During product design, it should always be ensured that the product is manufactured in an uncomplicated way, easy to repair or maintain and can be dismantled without problems at the end of its service life. The production of the future has a modular design and is automated to a considerable extent, so that system utilisation is optimal and the wastage quota is minimised. An entrepreneur should orient himself to this vision when he is thinking about the further development of his company.

Competitive advantages are generated in two respects through resource efficiency usage. Firstly, it is generated directly by when the use of materials goes hand in hand with lower material cost. Secondly, and indirectly, when a resource-efficient method of production communicates environmental advantages to the customer.

\section{Illustrative case studies}

Effizienz-Agentur NRW carries out about 250 projects per annum for increasing resource efficiency in small and medium-sized industry and trade companies in North Rhine-Westphalia, and also accompanies implementation. In parallel to the rapid increase in the importance of digitalisation for manufacturing companies, which has been discussed in Germany under the term 'Industry 4.0', the consultancy work of the Duisburg agency is also on the increase in this area. Based on the following practical examples, it is easy to understand the significant contribution that elements of digitalisation can make to resource efficiency, i.e. material and energy savings. It is not a case of creating a 'smart factory'. Particularly companies that operate in the value creation chain as suppliers and semi-finished product manufacturers, for example, and are subject to strict customer specifications often only have a limited amount of influence on changes. As far as a consultancy is concerned, here it is a case of finding the correct starting points and making use of digitalisation in locations where it is sensible and useful. 


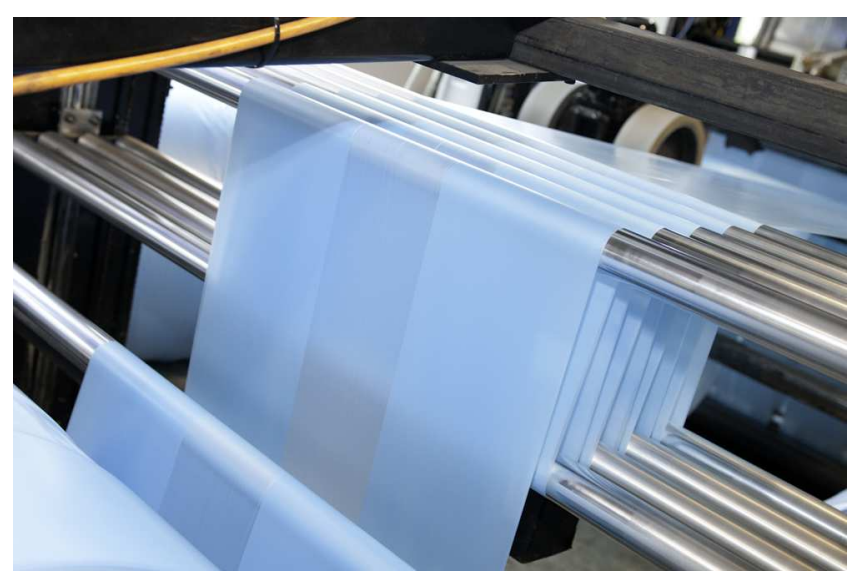

Figure 20.3 mk Plast GmbH \& Co KG processes more than 20 tons of plastic per day into plastic films.

Photo: Firma some.oner; Yashar Khosravani.

The three following consultancy cases or practical examples which are described in more detail in the following have been proportionally supported within the scope of the EFRE 'Resource Efficiency' consultancy programme.

Practical example 1 (industry: plastics)

\section{INITIAL SITUATION}

The core business of the family-run mk Plast GmbH \& Co. KG from the North Rhine-Westphalian town of Monschau is the manufacturing and processing of plastic film which is depicted in Figure 20.3.

Approximately 40 employees work at the location. More than 20 tons of plastic is processed per day with eight production machines (so-called blown film extruders) and production lines. Among other things, the film is used in the form of pouches and sacks in the packing industry and in medical engineering. To increase its process transparency and improve resource efficiency, the company used the resource efficiency consulting of Effizienz-Agentur NRW.

\section{RESULTS AND ADVANTAGES}

The process analysis showed that the operational connection of the machine park to the current information systems (Management Executive System MES, Enterprise Resource Planning ERP and Monitoring) and the evaluations thereof did not fulfil the increased demands that were being made concerning order calculation and organisation. 
The first step of the consulting, therefore concentrated on the order-specific maintenance systems and their connection to the operational data recording. The data that was collected constituted the prerequisite for providing the existing ERP system with the necessary operational information. The relevant process information such as compressed air flow rate, switch cabinet temperature, flow and return temperatures of the cooling water, total electrical output, melt pressure, cylinder temperature, diverter temperature and melting temperature as well as traction and melt throughput was first recorded on a pilot extruder, stored centrally in the database and then made available to the ERP system for evaluation. A particular challenge was interpreting and technically qualifying the complex variety of data in the sense of making more efficient use of resources.

Another goal of the consulting project was to reduce non-productive setup and downtimes during order changes on the blown film extrusion lines. Setup times and the start and completion of orders can now be accurately defined with the aid of automated machine data recording, and expenditure which is incurred can be documented and evaluated in detail. The setup times at the pilot extruder were able to be reduced by up to approx. 20 hours per year by these measures.

It was accompanied by wastage minimisation of approximately four tons of granules per annum with a throughput of approximately 200 kilograms per hour. At the same time, the productivity of the overall process was improved. Approximately 20 tons of waste production per annum will be saved in future when applied to all eight production lines. The company also expects a further reduction in film wastage of 30 tons per annum by making operative improvements to the cooling section of the blown film extrusion line. In total, mk Plast will save approximately 50 tons of polyethylene thanks to having more efficient process data recording and evaluation. As a result of the measures which have been implemented, including increased value creation and resource productivity, the company is anticipating an annual cost reduction of around 98,000 euros.

\section{Practical example 2 (industry: metal)}

\section{BACKGROUND AND GOALS}

Putzier Oberflächentechnik GmbH refines sections of steel using the thermal spraying process for mechanical engineering, the paper and electronics industries, and also the food processing industry, among others. Figure 20.4 illustrates how Putzier refines steel parts using thermal spraying processes.

The company from Leichlingen in North-Rhine Westphalia, which has around 40 employees, processes approximately 90 tons of steel and four tons of spray powder per annum. To improve resource efficiency, Putzier used the resource efficiency consulting of Effizienz-Agentur NRW. An initial analysis showed that the operational information systems (Enterprise Resource 

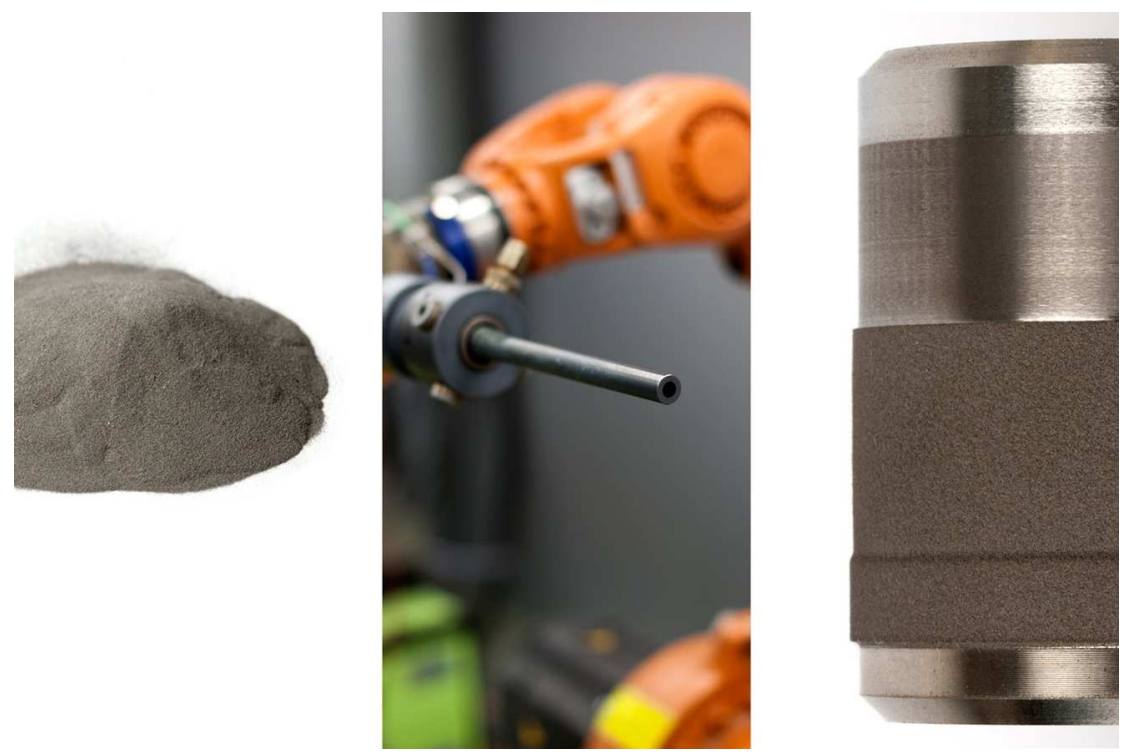

Figure 20.4 Refining of steel parts using thermal spraying processes by Putzier. Photo: Jens Putzier.

Planning ERP, production planning and control system PPS, operational data recording $\mathrm{BDE}$ ) and the evaluations from these systems had not been adapted to the increased requirements. To increase potential in this area, the company used one of the Excel tools developed by Effizienz-Agentur NRW for causeoriented assignment of resource costs (Ressourcenkostenrechnung, RKR, Resource Cost Accounting).

\section{RESULTS AND ADVANTAGES}

In the first stage of the consulting, the main focus was on the start-up and auxiliary processing times of the entire value creation and BDE data recording. The analyses were based on structured employee interviews. The results formed the basis for checking the ERP data management system. With the aid of resource cost accounting, the order flow, the handover of the order at the sales side, and also the planning of the orders in the manufacturing system were improved during the next stage. The purchasing processes are now more strongly linked to the order situation. The updating of the cost calculation (pre- and post-calculation) allows Putzier to analyse the cost-effectiveness of the order spectrum continually.

Orders which are uneconomical can now be quickly identified. The time taken for internal organisation, from the technical inquiry to the quotation calculation and the manufacturing order, was reduced by up to 60 per cent 
after the restructuring. Wastage quotas, internal complaints and stock levels were also reduced, and backlogs were also relieved. The reductions led to annual material savings of 2.4 tons and a three per cent reduction of the material quota within the company.

For Putzier, the process transparency that was gained has opened up an entirely new perspective for the further development of its corporate strategy. Moreover, the first stage of the digital transformation is complete. Other projects for the filling in gaps in the digitalisation are being planned. With the aid of dynamic project management, the company wants to align itself for the future, initiate the generation change and sustainably align its range of products to resource efficiency and Industry 4.0.

\section{Practical example 3 (industry: metal)}

\section{BACKGROUND AND GOALS}

Stainless steel factory Schmees manufactures high quality cast steel components at its NRW location in Langenfeld for the food and pharmaceutical industries and the mechanical energy engineering. Every year, Edelstahlwerke Schmees processes approximately 2,200 tons of steel into castings (see Figure 20.5).

Approximately 140 employees work at the Langenfeld location. A large number of product variants continuously presents the company with significant organisational challenges. Up until the beginning of 2016, the individual production areas looked after the manufacturing of the orders single-handedly. An initial analysis revealed that redundancies were occurring time and time again in the warehousing area, among others. To organise manufacturing and warehousing more transparently and efficiently, Schmees used Resource Cost Accounting

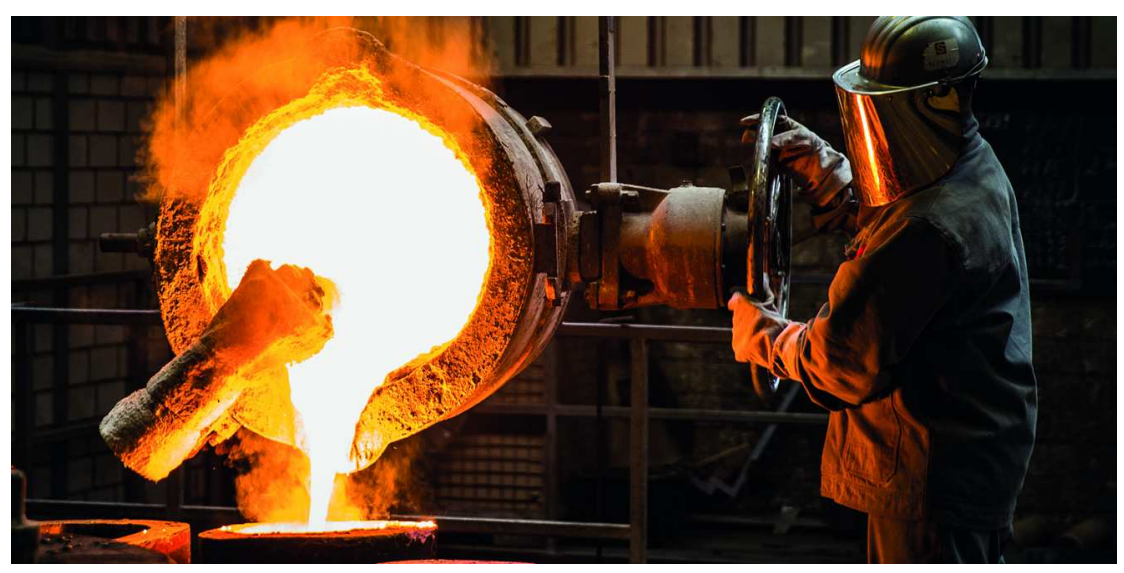

Figure 20.5 The Edelstahlwerke Schmees processes approximately 2,200 t of steel into castings. Photo: Edelstahlwerke Schmees GmbH. 
(Ressourcenkostenrechnung, RKR) from Effizienz-Agentur NRW as part of resource efficiency consulting. A created the data basis for a new module in the ERP system, was, thus created.

\section{RESULTS AND ADVANTAGES}

In 2015, with the help of RKR, Schmees recorded materials and energy usage in its cost structures. Based on the data that was collected, a cause-oriented cost accounting system was developed. This made it possible to evaluate individual orders in accordance with specific resource requirements.

The integration of RKR in the new ERP system has now put the company in a position to systematically analyse specific order groups such as rings and housings with regard to their material requirements, compare them and therefore determine the maximum material efficiency potential for each product via time series. Further, the integration of RKR in the new ERP system makes it possible to identify material wasters at an early stage.

This evaluation now constitutes the basis for the central planning and control of the material flow. With the aid of achieved new coordination options, the effectiveness of the manufacturing process was improved in such a way that the timeliness of order processing increased significantly. Also, there was a considerable reduction in the amount of reworking. The entire order process is now controlled centrally via production data acquisition. Schemes also set up a continuous improvement process, meaning that the flow of orders is getting better all the time.

These measures have resulted in a sustainable reduction in the number of unfinished castings and scrap quantities in production. The ERP system which has been supplemented by RKR is an essential step towards further digital networking of its processes for the company. Schmees invested approximately 10,000 euros in the development and implementation of the ERP solution and reduced its material costs by around 400,000 euros per annum.

\section{Summary}

Digitalisation fundamentally influences and changes how manufacturing companies produce their products. The bandwidth ranges from simple changes and improvements to operational processes to upheavals which cast doubt upon existing business models and make strategic engagement with the market and the future essential. On the one hand, the market influences products and production samples at increasing speed and in increasingly short cycles by means of digitalisation, and on the other hand the future viability of many manufacturing companies depends on how sensibly they make use of the elements of digitalisation in order to be able to benefit from the advantages thereof.

If you examine the proportion of material costs of about 42 per cent in the manufacturing industry in Germany, it becomes clear how much potential 
digitalisation has in the material and energy-saving areas, which have a decisive influence on a company's competitiveness. For the purpose of clarification, we can also talk about 'Resource Efficiency 4.0'. The practical examples reveal the concrete savings which can be made in terms of resources and emissions and also costs due to elements of digitalisation, i.e. using Resource Efficiency 4.0. The experience gained during the work of Effizienz-Agentur NRW has shown that dividing up into the elements of order clarification, order processing, calculation and company future in the analysis for evaluating Resource Efficiency 4.0 is useful for identifying weaknesses and initiating improvements accordingly using digitalisation instruments.

The examples also show that the prerequisite for the effective introduction and use of Resource Efficiency 4.0 is exact knowledge of your existing processes. An exact analysis of the relevant processes within the company is needed to do this to provide transparency. Only then can it be seriously decided whether and to what extent elements of digitalisation are to be introduced in the sense of Resource Efficiency 4.0. However, particularly small and medium-sized companies from industry and trade often lack the financial resources, the time and the relevant personnel to carry out appropriate analyses. Neutral and independent external consultancy can help in these cases, such as that which is provided by the Effizienz-Agentur NRW, which works on behalf of the North Rhine-Westphalia Ministry for the Environment.

\section{References}

Bliesner, A., Liedtke, C. \& Rohn, H. (2014). Resource efficiency and culture - Workplace training for small and medium-sized enterprises. Science of the Total Environment, 481, 645-648.

Bundesregierung, D. (2016). Deutsche Nachhaltigkeitsstrategie: Neuauflage 2016. Die Bundesregierung: Berlin, Germany.

European Environment Agency. (2016). More from Less-Material Resource Efficiency in Europe: 2015 Overview of Policies, Instruments and Targets in 32 Countries. Publications Office of the European Union.

Jahns, P. (2018). Protect Resources, Strengthen the Economy: Good Examples for Resource Efficiency in Industry and Handicraft Businesses. In Factor X (pp. 385-393). Springer, Cham.

Kaiser, R. (2018). Germany's Resource Efficiency Agenda: Driving Momentum on the National Level and Beyond. In Factor X (pp. 213-232). Springer, Cham.

Müller, J. M., Buliga, O. \& Voigt, K. I. (2018). Fortune favors the prepared: How SMEs approach business model innovations in Industry 4.0. Technological Forecasting and Social Change, 132, 2-17.

Müller, M. \& Reutter, O. (2017). Vision development towards a sustainable North RhineWestphalia 2030 in a science-practice-dialogue. Sustainability, 9(7), 1111. 